\title{
THE STAR FORMATION HISTORY AND MASS FUNCTION OF THE DOUBLE CLUSTER h AND $\chi$ PERSEI
}

\author{
CATHerine L. Slesnick ${ }^{1}$ and Lynne A. Hillenbrand ${ }^{1}$ \\ Department of Astronomy, MS 105-24, California Institute of Technology, Pasadena, CA 91125; \\ cls@astro.caltech.edu,lah@astro.caltech.edu \\ AND \\ PHILIP MASSEY ${ }^{1}$ \\ Lowell Observatory, 1400 West Mars Hill Road, Flagstaff, AZ 86001; phil.massey@lowell.edu \\ Received 2002 February 27; accepted 2002 May 18
}

\begin{abstract}
The $\mathrm{h}$ and $\chi$ Per "double cluster" is examined using wide-field $(0.98 \times 0.98)$ CCD UBV imaging supplemented by optical spectra of several hundred of the brightest stars. Restricting our analysis to near the cluster nuclei, we find identical reddenings $[E(B-V)=0.56 \pm 0.01]$, distance moduli (11.85 \pm 0.05$)$, and ages $(12.8 \pm 1.0 \mathrm{Myr})$ for the two clusters. In addition, we find an initial mass function slope for each of the cluster nuclei that is quite normal for high-mass stars, $\Gamma=-1.3 \pm 0.2$, indistinguishable from a Salpeter value. We derive masses of $3700 M_{\odot}(\mathrm{h})$ and $2800 M_{\odot}(\chi)$ integrating the present-day mass function from 1 to $120 M_{\odot}$. There is evidence of mild mass segregation within the cluster cores. Our data are consistent with the stars having formed at a single epoch; claims to the contrary are very likely due to the inclusion of the substantial population of early-type stars located at similar distances in the Perseus spiral arm, in addition to contamination by $\mathrm{G}$ and $\mathrm{K}$ giants at various distances. We discuss the uniqueness of the double cluster, citing other examples of such structures in the literature but concluding that the nearly identical nature of the two cluster cores is unusual. We fail to settle the long-standing controversy regarding whether or not the double cluster is the core of the Per OB1 association and argue that this may be unanswerable with current techniques. We also emphasize the need for further work on the pre-main-sequence population of this nearby and highly interesting region.

Subject headings: open clusters and associations: individual (NGC 869, NGC 884) — stars: early-type stars: luminosity function, mass function

On-line material: machine-readable tables
\end{abstract}

\section{INTRODUCTION}

The "double cluster" $\mathrm{h}$ and $\chi$ Persei (hereafter $\mathrm{h} / \chi$ Per; also known as NGC 869 and NGC 884, respectively) is among the brightest, densest, and closest of the open clusters containing moderately massive stars. The double cluster has been studied extensively over the last century (e.g., Oosterhoff 1937; Bidelman 1943; Wildey 1964; Schild 1965, 1967; Crawford, Glaspey, \& Perry 1970; Vogt 1971; Tapia et al. 1984; Waelkens et al. 1990) with resulting mean reddenings of $E(B-V)=0.5-0.6$ and distance moduli in the range 11.4-12.0 mag (1.9-2.5 kpc). The clusters contain several tens of Be stars (e.g., Slettebak 1968; Bidelman 1947a; see also Keller et al. 2001). Wildey's (1964) H-R diagrams (HRDs) suggested several distinct episodes of star formation (7, 17, and $60 \mathrm{Myr}$ ), which would imply a spread of more than $50 \mathrm{Myr}$ in the formation times of OB stars in a single (double) cluster! This age spread is larger than that claimed for any other well-studied open cluster and is one of the primary motivations of the present investigation.

Most previous work on $\mathrm{h} / \chi$ Per has used photographic or single-channel photoelectric photometry with little emphasis on spectroscopy. Several very recent papers have

\footnotetext{
${ }^{1}$ Visiting astronomer, Kitt Peak National Observatory, a division of the National Optical Astronomy Observatory, which is operated by the Association of Universities for Research in Astronomy, Inc., under cooperative agreement with the National Science Foundation.
}

used CCDs but consisted of photometric analysis only $(U B V I / \mathrm{H} \alpha$, Keller et al. 2001; ubvy $/ \mathrm{H} \beta$, Marco \& Bernabeu 2001). Distance moduli in the range 11.6-11.8 mag and ages of 10-20 Myr have been found, with Marco \& Bernabeu (2001) arguing (like Wildey 1964) for three distinct episodes of star formation, while Keller et al. (2001) find instead a single age. There is significant disagreement between various authors as to whether the reddenings, distances, and ages of the two clusters are identical or substantially $(30 \%-50 \%)$ different. It is especially important to understand in detail the star formation history of $h / \chi$ Per since these clusters are widely used from professional review papers to basic introductory astronomy textbooks to illustrate upper main-sequence stellar evolution.

Our modern study of $\mathrm{h} / \chi$ Per consists of wide-field CCD $U B V$ photometry for 4528 stars and blue optical spectroscopy for 196 of the stars presumed to be the most massive (i.e., the brightest blue and red stars). Our main goals are to redetermine the distance, age, and age spread in the double clusters and to explore for the first time the mass function and the evidence for mass segregation. In $\S 2$ we describe our data acquisition, reduction, and preliminary photometric and spectroscopic analysis. In $\S 3$ we present color-magnitude diagrams (CMDs), an assessment of field star contamination, a derivation of reddening and distance, and a Hertzsprung-Russell diagram along with discussion of stellar age and mass distributions. Section 4 contains our discussion of the uniqueness of this double cluster and the 
relationship between it and the surrounding region. In $\S 5$ we summarize our results.

\section{OBSERVATIONS AND DATA REDUCTION}

\subsection{Photometry}

$U B V$ photometry was obtained from observations with the $0.9 \mathrm{~m}$ telescope at Kitt Peak National Observatory using the Mosaic CCD camera (0".43 pixel $\left.^{-1}\right)$ on 1999 February 3. Conditions were photometric with $\sim 1$ !"3 seeing. The Mosaic camera consists of eight individual SITe $2048 \times 4096$ CCD chips arranged in two rows of four to produce a final image equivalent to 8192 pixels $\times 8192$ pixels $\left(0^{\circ} .98 \times 0^{\circ} .98\right)$ but with modest (35-50 pixel) gaps. Our imaging data set contains short ( $0.5 \mathrm{~s}$ in $V$ and $B$ and $2 \mathrm{~s}$ in $U$ ), medium ( $2 \mathrm{~s}$ in $V$ and $B$ and $10 \mathrm{~s}$ in $U$ ), and long ( $100 \mathrm{~s}$ in $V$ and $B$ and $300 \mathrm{~s}$ in $U$ ) integrations, each consisting of five dithered exposures that were combined to fill in gaps between the eight chips. The exceptions to this pattern are the short exposures, which were not dithered, and the medium $B$ exposure, which had only four dithers instead of five. Many Landolt (1992) standards were observed for the purposes of transformation to the $U B V$ system.

For the basic reductions we followed the precepts of Valdes (1998), using the IRAF "mscred" package. With bright twilight flats we were able to flatten the data to less than $1 \%$ in terms of large-scale gradients. Since the plate scale changes significantly over the field of view, care must be taken to geometrically correct the data to a uniform sampling for the premise of aperture photometry to work; this transformation was made using a sinc interpolation in order to come close to preserving the Poisson noise characteristics.

A common but hitherto untested practice when working with Mosaic data is to combine each set of ditherings into a single "stacked" image for photometry. However, each chip has its own spectral response and hence color term. Since any given star may be the average of multiple dithered exposures and may appear on up to four of the CCDs, we were driven to wonder to what degree of accuracy one could do photometry using the final combined images. We thus performed both aperture photometry and point-spread function (PSF) fitting photometry separately on both the stacked images ( 9 total $=3$ colors $\times 3$ integration times) and the individual CCD frames $(247$ total $=8$ chips $\times 1,4$, or 5 ditherings $\times 3$ colors $\times 3$ integration times). Color terms were determined for each of the eight chips individually. We retained the median value in each filter for the stacked images. On the whole, color-term variations were most significant at $U$. By adopting a single color term for the stacked images, we expect to make systematic errors in $V$ by an average of $0.012 \mathrm{mag}$ over a color range in $B-V$ of 1. The maximal chip-to-chip difference is $0.032 \mathrm{mag}$ in $V$. The $B$ band gave similar residuals with a full range of 0.038 per 1 mag range in $B-V$. In $U$ band the chip-to-chip offsets were considerably larger, yielding typical variations of 0.038 mag over a range of 1 mag in $U-B$ and a maximal difference of 0.11 mag.

We found that PSF photometry of the stacked images produces errors of greater than 5\%, independent of magnitude, suggesting that these errors are not dominated by photon noise. The scatter for the individual frame PSF photometry was also magnitude independent and gave errors of $\sim 3 \%$ when compared to aperture photometry of single isolated stars. We could see by visual inspection that there were significant variations in the PSF even across a single chip, despite the relatively slow (f/7.5) beam. ${ }^{2}$ Accordingly, we choose to rely on aperture photometry alone, sacrificing the potential advantage of PSF fitting for any crowded stars. Fortunately, $\mathrm{h} / \chi$ Per is relatively sparse. Over the entire imaging area, the mean stellar density to $V=15 \mathrm{mag}$ is 0.7 stars $\operatorname{arcmin}^{-2}$; in the center of the clusters, it is still a modest 2.2 and 1.7 stars $\operatorname{arcmin}^{-2}$ for $\mathrm{h}$ and $\chi$, respectively.

Our standard and program stars were each measured with the same large aperture (10 pixel radius $=8 . \prime 6$ diameter). The standard star data were then used to produce transformations between the instrumental and standard system. The extinction values that we found were typical of Kitt Peak, and our fits had small $(<0.02 \mathrm{mag})$ residuals.

Because our frames went much deeper than any program stars of interest (thanks to the long-exposure frames), we had the luxury of retaining only the very best data for the subsequent analysis. After merging the data for the three sets of exposures times, we kept only those stars for which the instrumental errors (due to photon statistics and read noise) were less than $0.01 \mathrm{mag}$ in $U, B$, and $V$. This eliminated roughly $96 \%$ of the stars that we had measured and is equivalent to simply imposing a magnitude cutoff on the data. Thus, while our catalog does not go as deep as other recent efforts (see Keller et al. 2001), our photometric errors are quite small, and we purposefully chose to truncate our catalog once field contamination became extreme.

Table 1 contains our catalog of $h / \chi$ Per stars, ordered by decreasing brightness. We have merged the photometry for the three sets of exposures, weighting inversely by the square of the photometric uncertainty. Our final source list contains three-filter photometry for 4528 stars down to $V \sim 16$ mag. We can estimate our completeness from the histograms of the number of stars per $0.25 \mathrm{mag}$ bin shown in Figure 1 and find completeness of our catalog to $U \sim 16.2$, $B \sim 16.0$, and $V \sim 15.0$.

How well does our photometry agree with earlier studies? In Figure 2 we compare our work with a subset of Wildey's (1964) photoelectric and photographic work, concentrating primarily on the brighter stars and those for which we have spectral types. We see that his photometry and ours agree extremely well given the differences in equipment. We find differences (in the sense of our data minus Wildey's) from his photographic data of $(\Delta V)_{\text {avg }}=0.097 \pm 0.027$, $[\Delta(B-V)]_{\mathrm{avg}}=-0.044 \pm 0.009$, and $\quad[\Delta(U-B)]_{\mathrm{avg}}=$ $-0.069 \pm 0.036$ computed from a comparison of 300 stars. Comparing our photometry to 24 of Wildey's photoelectrically observed stars, we find even smaller average offsets of $(\Delta V)_{\mathrm{avg}}=0.020 \pm 0.022, \quad[\Delta(B-V)]_{\mathrm{avg}}=-0.014 \pm 0.026$, and $[\Delta(U-B)]_{\mathrm{avg}}=0.017 \pm 0.012$. The scatter is larger and asymmetrical in the $V$ comparison (see Fig. 2), in the sense one would expect if Wildey's work occasionally had faint stars in the sky determination. In a similar comparison with Wildey's photographic study, Keller et al. (2001) quote average differences of $(\Delta V)_{\text {avg }}=0.16, \quad[\Delta(B-V)]_{\text {avg }}=$ -0.03 , and $[\Delta(U-B)]_{\mathrm{avg}}=0.00$, attributing the offset with respect to Wildey's $V$-band photometry to crowding effects.

\footnotetext{
${ }^{2}$ Subsequent to these data being obtained, the corrector in the $0.9 \mathrm{~m}$ was realigned, leading to improved behavior of the PSF.
} 
TABLE 1

OBSERVATIONAL Data

\begin{tabular}{|c|c|c|c|c|c|c|c|c|c|c|c|}
\hline \multirow[b]{2}{*}{ ID } & \multirow{2}{*}{$\begin{array}{c}\alpha \\
(\mathrm{J} 2000.0)\end{array}$} & \multirow{2}{*}{$\begin{array}{c}\delta \\
(\mathrm{J} 2000.0)\end{array}$} & \multirow[b]{2}{*}{$V$} & \multirow[b]{2}{*}{$B-V$} & \multirow[b]{2}{*}{$U-B$} & \multirow[b]{2}{*}{$d_{\mathrm{h}}^{\mathrm{a}}$} & \multirow[b]{2}{*}{$d_{\chi}^{\mathrm{a}}$} & \multirow[b]{2}{*}{ Assoc. } & \multicolumn{2}{|c|}{ SPECTRAL Type } & \multirow[b]{2}{*}{ Alt. ID ${ }^{d}$} \\
\hline & & & & & & & & & Adopted $^{b}$ & Literature $^{\mathrm{c}}$ & \\
\hline $1 \ldots \ldots \ldots \ldots$ & 21804.44 & 573058.9 & 6.023 & 1.097 & 0.832 & 0.389 & 0.681 & far & G7 III* & G7 III (4) & HD 13994 \\
\hline $2 \ldots \ldots \ldots \ldots$ & 22155.32 & 571434.6 & 6.480 & 0.502 & -0.050 & 0.403 & 0.129 & far & $\mathrm{A} 0 \mathrm{I}$ & A1 Ia (2) & HD 14433 \\
\hline 3................... & 21904.37 & 570808.4 & 6.567 & 0.452 & -0.346 & 0.016 & 0.425 & $\mathrm{~h}$ & B3 I & B3 Ia (1) & HD 14134 \\
\hline $4 \ldots \ldots \ldots \ldots$ & 21913.86 & 571009.8 & 6.700 & 0.503 & -0.428 & 0.035 & 0.405 & $\mathrm{~h}$ & B3 I & B2 Ia (1) & HD 14143 \\
\hline $5 \ldots \ldots \ldots \ldots$ & 22300.35 & 572313.5 & 6.977 & 0.707 & -0.249 & 0.587 & 0.288 & far & B8 I & B8 Ia $(2,3)$ & HD 14542 \\
\hline . . . . . . . & $\ldots$ & $\ldots$ & $\ldots$ & $\ldots$ & $\ldots$ & $\ldots$ & $\ldots$ & $\ldots$ & $\ldots$ & $\ldots$ & $\ldots$ \\
\hline $4528 \ldots \ldots$. & 21935.40 & 571829.9 & 16.906 & 0.160 & -0.609 & 0.176 & 0.400 & far & $\ldots$ & $\ldots$ & $\ldots$ \\
\hline
\end{tabular}

NoTES.-Units of right ascension are hours, minutes, and seconds, and units of declination are degrees, arcminutes, and arcseconds. Table 1 is published in its entirety in the electronic edition of the Astrophysical Journal. A portion is shown here for guidance regarding its form and content.

${ }^{\text {a }}$ Distances from the cluster centers are given in degrees.

${ }^{\mathrm{b}}$ An asterisk $(*)$ denotes that we have used a spectral type from the literature in our analysis.

c References: (1) Schild 1965; (2) Johnson \& Morgan 1955; (3) Slettlebak 1968; (4) Appenzeller 1967; (5) Bidelman 1947b; (6) Morgan, Code, \& Whitford 1955 .

${ }^{\mathrm{d}}$ Our cross-identifications are not complete and focus on the brighter stars and those for which we have spectral types.

We have matched our data to that of Keller et al. (2001) for stars that are not known from the literature to be variable and for which we have identified Oosterhoff (1937) numbers (again, our cross-identification is not complete) and find average offsets of $(\Delta V)_{\mathrm{avg}}=-0.019 \pm 0.008$, $[\Delta(B-V)]_{\mathrm{avg}}=0.001 \pm 0.026$, and $[\Delta(U-B)]_{\mathrm{avg}}=-0.048$ \pm 0.014 computed from 55,31 , and 49 stars, respectively. The generally good agreement between all three studies is a testament to Wildey's painstaking accuracy in centering stars with a photoelectric photometer and also supports the validity of our reduction procedure and transformation to the standard photometric system.

\subsection{Spectroscopy}

Several hundred spectral types complement our photometric database. For hot stars, spectral data are needed to obtain accurate effective temperatures and consequently accurate extinction estimates and bolometric corrections (Massey 1998a, 1998b), all necessary for locating a star in the H-R diagram. We selected stars for spectroscopy based on their brightness and colors. Since we did not yet have our own CCD photometry at the time the spectroscopic program was begun, we worked largely from the Wildey (1964) photometry; this introduces a bias toward blue stars closer to the cluster nuclei. Later spectroscopic runs incorporated a wider range of magnitude and color selection, probing down as far as mid-A spectral types in an unrealized attempt to identify possible pre-main-sequence stars amidst substantial field star contamination. In the lower left panel of Figure 4 we show the spatial distribution of the spectroscopic sample compared to the entire photometric sample; in the upper left panel we show the loci of the spectroscopic sample in the color-magnitude plane. Of the brightest 50 stars, we have spectral types for 49 , regardless of location in our field. Fainter than that, the spectroscopic campaign was concentrated in the cluster cores.

Spectroscopic data were taken at several NOAO telescopes. We employed the WIYN $3.5 \mathrm{~m}$ telescope and the Hydra multifiber positioner to feed a bench-mounted spectrograph (1993 December and 1994 October), the KPNO 4 $\mathrm{m}$ telescope and the $\mathrm{RC}$ spectrograph in multislit mode
(1994 November and 1999 August), the KPNO $2.1 \mathrm{~m}$ telescope and GoldCam with a single slit (1994 September), and the coudé feed telescope (1999 July and November). For most of the spectra the spectral range is $\sim \lambda \lambda 3900-4700$ at a resolution of $\sim 1.5 \AA$. Higher resolution was obtained with the coudé feed data, which were taken in multiple wavelength settings. One-dimensional spectra were extracted from the two-dimensional images using the slit and multifiber reduction packages within IRAF. Signal-to-noise ratios ranged from $\sim 20-150$ with nearly all spectra classifiable. Figure 3 shows three spectra taken at the KPNO $2.1 \mathrm{~m}$ telescope with GoldCam that illustrate the effects of temperature on B-type supergiants.

We present in Table 1 new spectral types for 196 stars, many of which result from several different observations of the same object. In classifying the spectra we followed the guidelines of Walborn \& Fitzpatrick (1990), Jaschek \& Jaschek (1987), and Jacoby, Hunter, \& Christian (1984). All stars were classified by a minimum of two of the authors, both independently and collaboratively. Spectral types assigned by us were also compared to those in the literature where available, and we cite these older spectral types as well. As emphasized in the introduction, spectroscopic efforts have lagged behind photometric studies of this region. Johnson \& Morgan (1955), Schild (1965, 1967), and Slettebak (1968) have made the most systematic efforts in this regard, and in general our spectral types agree very well with theirs.

The most luminous stars that we identify in the vicinity of $\mathrm{h} / \chi$ Per are M, A, and B supergiants. There is a lone O-type star, HD 14434 (O6.5 V). As we discuss below, this star is likely not a member of the double cluster but appears to be a younger field star interloper at approximately the same distance. The remainder of the spectra are slightly evolved B-type giants and B- and A-type dwarfs. We identify $10 \mathrm{Be}$ stars, two of which were previously unknown. Six out of seven stars that were classified photometrically as Be stars by Keller et al. (2001) using $(V-\mathrm{H} \alpha)$ colors and that we have our own spectra for do in fact prove to be emission-line objects. Since none of our spectra extend as far redward as $\mathrm{H} \alpha$, emission seen by us is usually in $\mathrm{H} \beta$, which tends to be weaker than $\mathrm{H} \alpha$ emission by about one-third. 

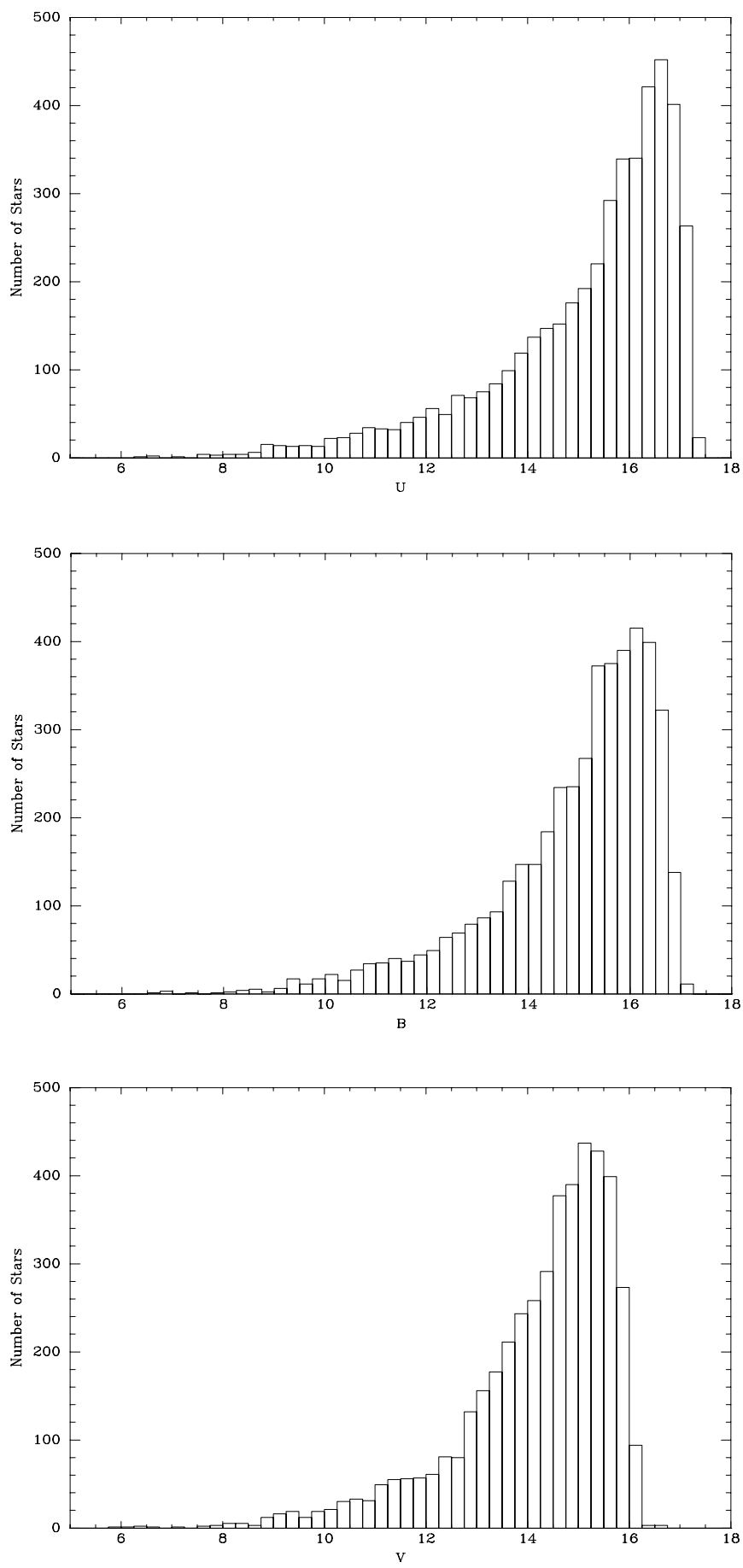

FIG. 1.-For each of our three filters, we use the histogram of the number of stars as a function of magnitude to estimate our completeness.

\section{ANALYSIS}

Our analysis includes discussion of color-magnitude diagrams, assessment of field star contamination, derivation of cluster reddening and distance, and construction of H-R diagrams. We then discuss stellar ages and masses as inferred from the $\mathrm{H}-\mathrm{R}$ diagrams and the distribution of ages and masses within the clusters.

We expect that stars near the cluster cores predominantly will be members, while stars further afield will be a mixture of both members and nonmembers. We constructed a contour plot of the spatial distribution of stars within our field and found that the stellar densities were enhanced by $2 \sigma$ at identical radii of $7^{\prime}$ from each of the cluster cores; we will use this radial criterion when describing stars near the nuclei. We also determined accurate centers for the two cores $\left(\alpha=2^{\mathrm{h}} 19^{\mathrm{m}} 22^{\mathrm{s}} \mathrm{s}, \delta=+57^{\circ} 09^{\prime} 00^{\prime \prime}[\mathrm{J} 2000.0]\right.$ for h Per, and $\alpha=2^{\mathrm{h}} 22^{\mathrm{m}} 12^{\mathrm{s}} \cdot 0, \delta=+57^{\circ} 07^{\prime} 12^{\prime \prime}$ [J2000.0] for $\chi$ Per) by examining mass and number density contours.

Tables $2 \mathrm{~A}$ and $2 \mathrm{~B}$ contain derived quantities for stars near $\left(\leq 7^{\prime}\right)$ and further away from the cluster centers, respectively. We have limited these tables to those stars that we included in determining the PDMF (see $\S 3.4 .4$ ).

\subsection{Color-Magnitude Diagrams and the Influence of Field Stars}

In Figure 4 we show the color-magnitude diagram for (1) all of the stars over the full $0^{\circ} .98 \times 0^{\circ} .98$ covered in our CCD images (left panels) and (2) only the stars within 7' of each of the two cluster nuclei (right panels). The influence of field stars can be seen in the upper left panel of this figure, notably between $0.5<B-V<1.0$ and $10<V<16$. For the $\mathrm{h}$ and $\chi$ cluster nuclei in the upper right panel, field star contamination is less severe but still present. The close match between the two CMDs suggests that there are no substantial differences in reddening, distance, or age between the two clusters, a conclusion we explore in greater depth below.

To further assess the field star contamination we use the density of stars in the color-magnitude diagrams (i.e., a "Hess" diagram), as shown in Figure 5 for both $V$ versus $B-V$ and $V$ versus $U-B$. We define the "cluster region" as above, while the "field stars" region is arbitrarily taken as the northern $0.25 \mathrm{deg}^{2}$ and southern $0.15 \mathrm{deg}^{2}$ of our CCD imaging area. Hess diagrams produced for this total area of $0.4 \mathrm{deg}^{2}$ were scaled up to the full area of the CCD survey (shown as red contours in the upper panels of Fig. 5) and subtracted from the Hess diagram constructed over the full imaging area. The result of this subtraction is shown in the lower panels, with the resulting $V$ versus $B-V$ and $V$ versus $U-B$ diagrams displaying a much tighter color-magnitude sequence than the full area.

\subsection{Reddening}

For each star with a spectral type, we compute the color excess using the spectral type-intrinsic color relations of FitzGerald (1970). The average value of color excess for 123 stars with well-determined spectral types is $E(B-V)=0.55$, with a $1 \sigma$ variation of 0.1 . For the 56 stars near the core of h Per, we find an average $E(B-V)=0.57$, with a $1 \sigma$ variation of 0.08 . Similarly, for 40 stars near the core of $\chi$ Per we find an average of $0.53(1 \sigma=0.08)$. The median values are $0.56,0.57$, and $0.55 \mathrm{mag}$ for the three samples, respectively. We conclude that the reddening is indistinguishable for the two clusters and, further, infer that the reddening is entirely due to line-of-sight extinction to the Perseus spiral arm with intracluster reddening essentially zero. This is consistent with a $3 \sigma$ upper limit on ${ }^{13} \mathrm{CO}$ emission (L. A. Hillenbrand \& J. M. Carpenter 1999, unpublished FCRAO data), corresponding to essentially no gas $\left[N\left({ }^{13} \mathrm{CO}\right)<2.1 \times 10^{15} \mathrm{~cm}^{-2}\right]$ or dust $\left(A_{V}<1.4 \mathrm{mag}\right)$ within the clusters.

In dereddening the photometry, we assume the standard 3.1 ratio of total-to-selective absorption. For other stars without spectral types, color excesses were determined using 

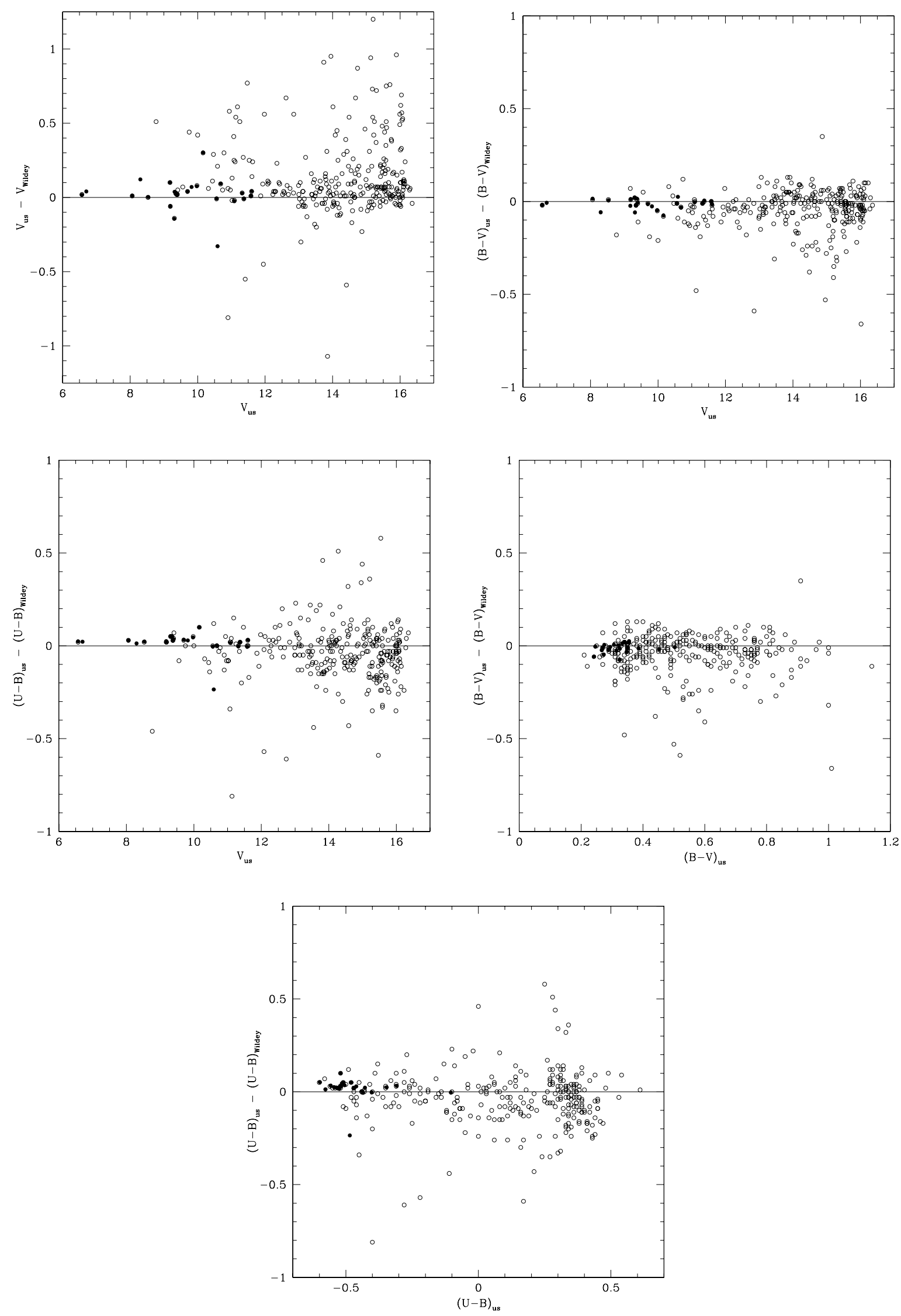

FIG. 2.- Our CCD photometry is found to agree well with that of Wildey's (1964) data. Open and filled circles are comparisons to his photographic and photoelectric photometry, respectively. 


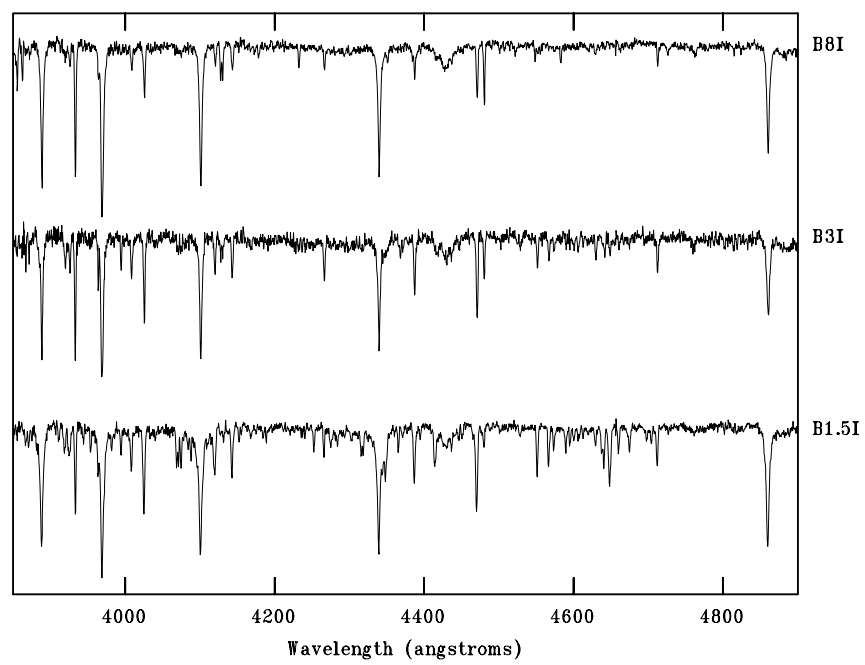

FIG. 3.-Temperature effects in B-type supergiants. The primary indicator is the ratio of $\mathrm{Mg}$ II 4481 to He I 4471.

the " $Q$-method," where applicable: following Massey (1998a), for stars with $B-V \leq 0.5$, we compute $(B-V)_{0}=-0.0186+0.3218 Q$, where

$$
Q=(U-B)-0.72(B-V)
$$

is a reddening-independent index. The relation between $(B-V)_{0}$ and $Q$ was derived by fitting the intrinsic color relationships of FitzGerald (1970) for main-sequence stars, but the relationship can be used for supergiants and giants. For redder stars without spectral types, we adopt the median $E(B-V)$ of 0.56 .

\subsection{Distance}

We determine the distances to the clusters using two methods: spectroscopic parallax and "main-sequence fitting." We discuss these two approaches separately and then comment on their respective merits.

\subsubsection{Spectroscopic Parallax}

For each star with a spectral type we compute the distance modulus by first dereddening the data using the intrinsic colors of FitzGerald (1970) and then finding $V_{0}-M_{V}$. We have adopted the spectral type $-M_{V}$ calibration of Conti

TABLE 2A

Derived Quantities for Probable Cluster Members

\begin{tabular}{ccccccc}
\hline \hline ID & HRD $^{\mathrm{a}}$ & $\log T_{\text {eff }}$ & $E(B-V)$ & $M_{V}$ & $M_{\text {bol }}$ & $\begin{array}{c}\text { Mass } \\
\left(M_{\odot}\right)\end{array}$ \\
\hline $3 \ldots \ldots \ldots$ & $\mathrm{s}$ & 4.300 & 0.58 & -7.09 & -9.09 & 33.1 \\
$4 \ldots \ldots \ldots$. & $\mathrm{s}$ & 4.300 & 0.63 & -7.11 & -9.11 & 33.1 \\
$9 \ldots \ldots \ldots$. & $\mathrm{s}$ & 4.385 & 0.57 & -5.57 & -7.99 & 21.0 \\
$12 \ldots \ldots$. & $\mathrm{s}$ & 4.370 & 0.42 & -4.86 & -7.24 & 16.4 \\
$16 \ldots \ldots$. & $\mathrm{s}$ & 4.340 & 0.56 & -5.12 & -7.34 & 16.8 \\
$\ldots \ldots \ldots$. & $\ldots$ & $\ldots$ & $\ldots$ & $\ldots$ & $\ldots$ & $\ldots$ \\
$1582 \ldots \ldots$ & $\mathrm{p}$ & 4.144 & 0.59 & 0.59 & -0.61 & 4.0 \\
\hline
\end{tabular}

Note.-Table 2A is published in its entirety in the electronic edition of the Astrophysical Journal. A portion is shown here for guidance regarding its form and content.

a This column indicates how the star was placed in the H-R diagram, with an "s" or " $p$ " meaning using spectra or just photometry, respectively. An " a" indicates that the mean $E(B-V)$ was adopted.
TABLE 2B

Derived Quantities for Probable Field Stars

\begin{tabular}{|c|c|c|c|c|c|c|}
\hline ID & $\mathrm{HRD}^{\mathrm{a}}$ & $\log T_{\text {eff }}$ & $E(B-V)$ & $M_{V}$ & $M_{\mathrm{bol}}$ & $\begin{array}{l}\text { Mass } \\
\left(M_{\odot}\right)\end{array}$ \\
\hline $1 \ldots \ldots \ldots$ & $\mathrm{s}$ & 3.680 & 0.16 & -6.31 & -6.68 & 13.7 \\
\hline $2 \ldots \ldots \ldots$ & $\mathrm{s}$ & 4.000 & 0.50 & -6.93 & -7.20 & 16.2 \\
\hline $5 \ldots \ldots \ldots$ & $\mathrm{s}$ & 4.050 & 0.73 & -7.13 & -7.71 & 18.8 \\
\hline $6 \ldots \ldots \ldots$ & $\mathrm{s}$ & 3.940 & 0.75 & -6.73 & -6.77 & 13.9 \\
\hline 7............... & $\mathrm{s}$ & 3.525 & 0.62 & -5.93 & -7.43 & 13.6 \\
\hline $1610 \ldots$. & $\cdots$ & 4.138 & 0.62 & 0.52 & -065 & 40 \\
\hline
\end{tabular}

Note.-Table 2B is published in its entirety in the electronic edition of the Astrophysical Journal. A portion is shown here for guidance regarding its form and content.

a This column indicates how the star was placed in the H-R diagram, with an "s" or "p" meaning using spectra or just photometry, respectively. An " a" indicates that the mean $E(B-V)$ was adopted.

(1988) for our one O-type star and that of Humphreys \& McElroy (1984) for everything else (B type through M type), interpolating values for spectral types not explicitly present in these tables when needed. For the stars with well-defined spectral types, we find a distance modulus of $12.5 \pm 0.5 \mathrm{mag}$ when we restrict ourselves to the objects within the cluster cores.

This method works relatively well for very young $(<3$ Myr) clusters (see Massey, Johnson, \& DeGioia-Eastwood 1995) where there is a fairly gradual change of $M_{V}$ with spectral type among the O-type stars (Conti 1988), about $1.4 \mathrm{mag}$ from $\mathrm{O} 3 \mathrm{~V}$ to $\mathrm{O} 9.5 \mathrm{~V}$. For a 10-20 Myr old cluster, the only stars left on the main sequence are B-type stars, and there $M_{V}$ changes by 3 mag over the spectral range $\mathrm{B} 0 \mathrm{~V}$ to B8 V. In addition, the luminosity criteria for B-type stars are rather subtle compared to those of the O-type stars; for the former it depends upon the absolute strengths of the $\mathrm{Si}$ lines (which are also temperature dependent), while for O-type stars it depends primarily on He II $\lambda 4686$ being in emission or absorption.

We did examine the spectroscopic parallaxes as a function of luminosity class. Although the median distance moduli for luminosity class V, III, and I stars are about the same, in each case there is a large spread in the values, which we attribute to errors in placing the stars into the correct luminosity class. Because of the subtleties involved, some misclassifications are inevitable, and in addition membership issues may also come into play. More significantly, stars that are really luminosity class IV and hence follow their own (and presently undetermined) spectral type $-M_{V}$ calibration are placed at present into either luminosity class III or class V. The same holds for luminosity class II stars, which often wind up being called either class I or class III because of lack of observational distinction between the classes.

Using only the brightest stars $(V<10.5)$, we find a distance modulus of $11.95 \pm 0.2$, in substantial agreement with what we find below. These brighter stars are among the earliest types yet include many supergiants whose intrinsic luminosity has a large scatter. However, the strength of the Si lines makes spectral classification more certain in classifying hot giant and supergiant stars. Therefore, spectral types and luminosity classes will be better determined for these bright stars. 

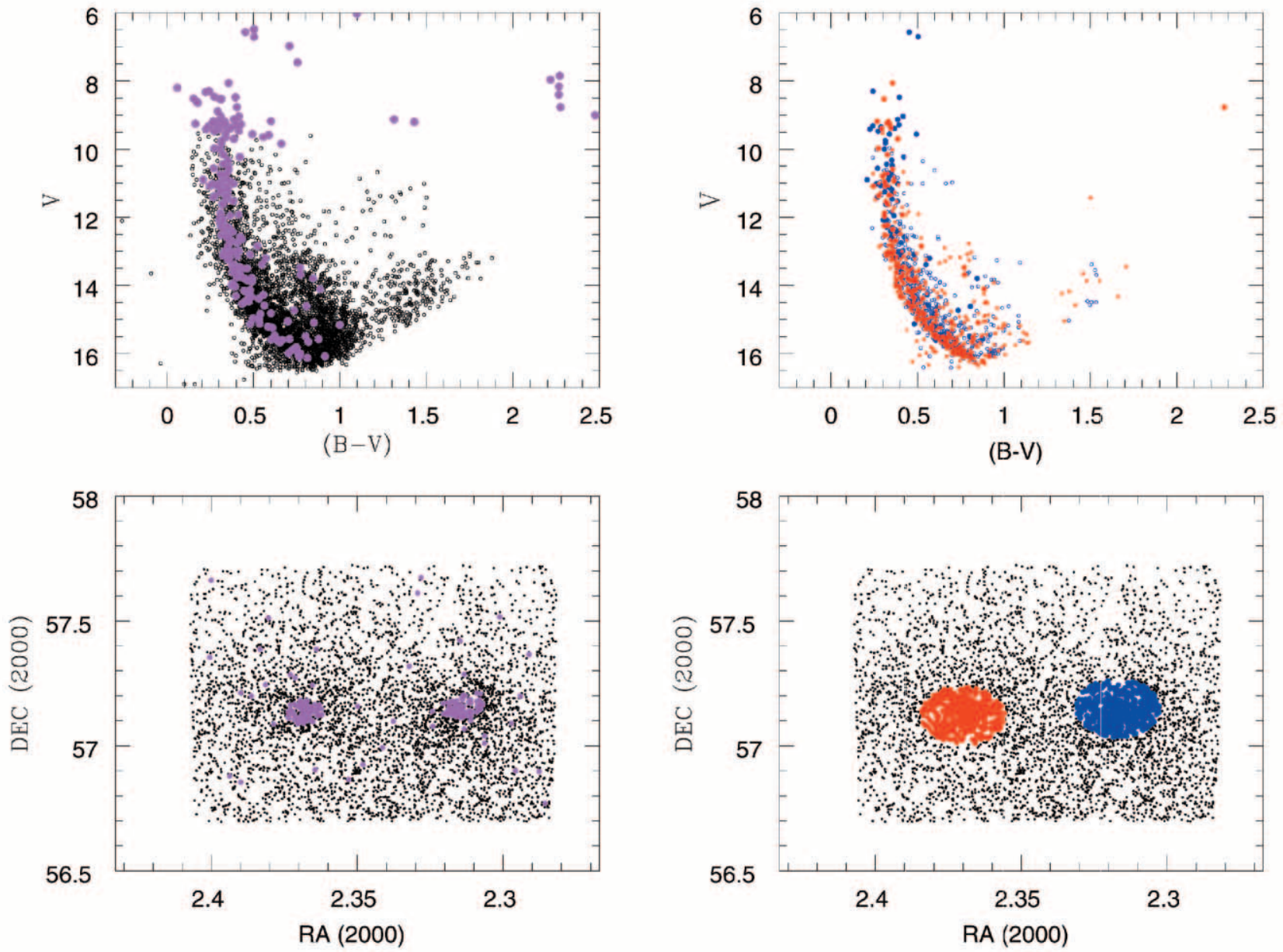

FIG. 4.-On the left we show the uncorrected CMD for all of the stars in our sample (upper panel), along with their spatial distribution (lower panel). We have overlaid colored symbols on the stars for which we have spectral types. These may be compared to the diagrams on the right, where we have included only stars within $7^{\prime}$ of the nuclei of the two clusters. Stars near the center of $h$ Per are indicated in blue, while stars near the center of $\chi$ Per are indicated in red.

\subsubsection{Main-Sequence Fitting}

We can also use "main-sequence fitting" to determine the average distance of the lower mass, unevolved stars in our sample. Given the approximate age of the clusters (13 Myr, as will be discussed in $\S 3.4 .3$ ), this is not a trivial exercise and must be done using post- and pre-main-sequence evolutionary tracks combined. Although many renditions of the "observed" main sequence exist (e.g., Balona \& Shobbrook 1984; Balona \& Feast 1975; Morton \& Adams 1968; Blaauw 1964), any relationship between $M_{V}$ and $(B-V)_{0}$ derived for stars in the solar vicinity necessarily contains only the mean $M_{V}$ value characteristic of the typical age in the solar neighborhood of stars with that $(B-V)_{0}$. For example, the $M_{V}$ values at the bluest values of $(B-V)_{0}$ represent ages of only 1-2 Myr, while the $M_{V}$ values around $(B-V)_{0}=0$ represent ages of a few hundred megayears and the $M_{V}$ values around the $(B-V)_{0}$ color of the Sun represent ages of more than a gigayear. In $\mathrm{h} / \chi$ Per, plotting the dereddened cluster data points against an observed main sequence results in the "main sequence" being too blue/ faint at the bluest colors (since the $\mathrm{h} / \chi$ Per stars are evolved away from the zero-age main sequence) and too bright/red at the redder colors (since the $\mathrm{h} / \chi$ Per stars are younger than the mean age of stars in the solar vicinity and, hence, not yet far enough evolved from the empirical zero-age main sequence to the position where stars having the mean age of the solar neighborhood would lie). Comparison to a theoretical zero-age main sequence involves similar concepts.

"Main-sequence fitting" therefore must be done when trying to fit more evolved clusters using theoretical isochrones. We use the solar metallicity $(Z=0.02)$ post-zeroage main sequence tracks and isochrones of Schaller et al. (1992), which include convective overshoot and standard mass-loss rates. In addition, we use the pre-main-sequence tracks and isochrones from the same group, published in Bernasconi (1996). We transform these tracks and isochrones from $M_{\text {bol }}$ and $\log T_{\text {eff }}$ to $V_{0}$ and $(B-V)_{0}$ using the same, though inverted, relationships that we use later to transform our data from the observational $V_{0}$ and $(B-V)_{0}$ plane to the theoretical $M_{\text {bol }}$ and $\log T_{\text {eff }}$ plane (see $\S 3.4$ ). Figure 6 is a CMD of our dereddened photometry where we have used the isochrones to determine the distance. As in Figure 4, blue and red points represent stars that are spatially located within $7^{\prime}$ from the centers of $h$ Per and $\chi$ Per, respectively.

The "main-sequence fitting" procedure is complicated by the fact that in order to obtain a best-fit distance modulus from theoretical isochrones an approximate age must be assumed. We explored isochrones spanning a wide range in 

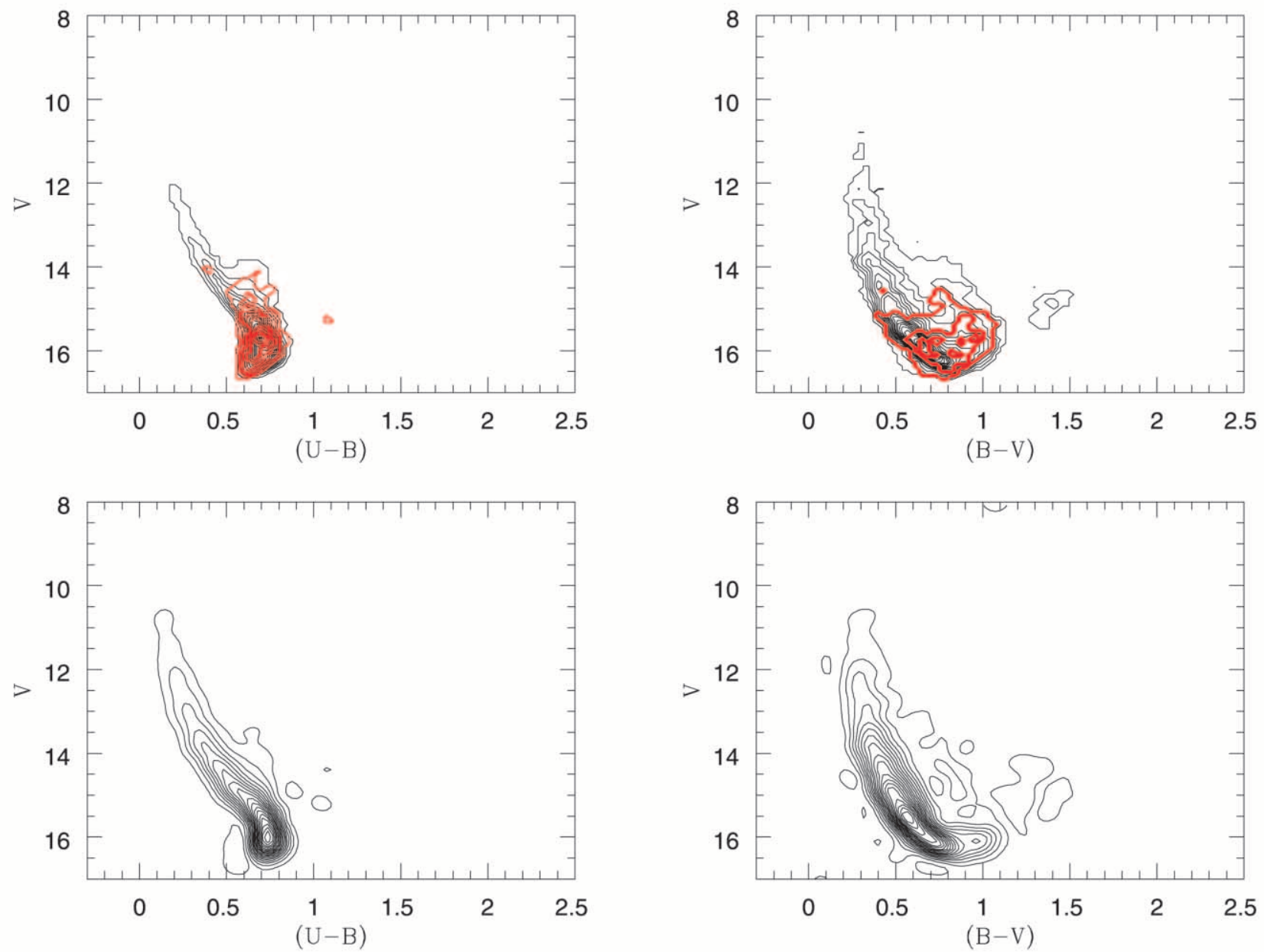

FIG. 5.-Top panels show the full CMDs plotted as contoured Hess diagrams, where we have overlaid color contours to indicate the field star contamination. In the bottom panels we have removed the field star contamination and smoothed the data.

age (1-50 Myr) and found that the isochrone shape matches the cluster data best for both post- and pre-main-sequence tracks in the 10-20 Myr range.

Using this information, we find a best-fitting distance modulus of $11.85 \pm 0.05$ mag corresponding to a distance of $2344_{-53}^{+55} \mathrm{pc}$, where we have estimated both the fit and uncertainty by eye in matching the models to the data.

Good agreement is seen between the post-zero-age main sequence turnoff at $(B-V)_{0} \leq-0.22$ and pre-zero-age main sequence turn-on at $(B-\bar{V})_{0} \geq 0.1$ using the Schaller et al. (1992) and Bernasconi (1996) calculations when transformed using our equations relating $\log T_{\text {eff }}$ to $(B-V)_{0}$ colors and bolometric corrections. Using transformations to the color-magnitude plane supplied directly by the authors (which rely upon the Schmidt-Kaler 1982 relationships) does not produce a match between the theory and the data for any isochrone. However, using our transformation equations (derived primarily from stellar atmosphere models), we find extremely good agreement between our dereddened data and the stellar evolutionary isochrones. As can be seen in Figure 6, at an age of 10-15 Myr, we expect to see significant contributions from the pre-main-sequence population at $M_{v} \sim 2$. However, because of the large amount of field star contamination is this region, the extent of this effect in our sample is difficult to determine.
Figure 6 effectively puts to rest any question as to whether or not $\mathrm{h}$ and $\chi$ Per are at two different distances rather than one. This result is supported by similar conclusions found by Keller et al. (2001).

Why are the distance moduli derived from spectroscopic parallax (12.5 mag) and photometric parallax (11.85 mag) so different? The slightly evolved state of the main-sequence stars that dominate our spectroscopic sample should actually lead to our computing too small a spectroscopic distance modulus rather than too great a number. We believe there is need for good recalibration of the spectral type-to- $M_{V}$ relation using a variety of clusters and associations with good distance moduli determined from spectroscopic parallax of O-type stars, as well as direct determinations via modern trigonometric parallaxes.

\subsection{The Hertzsprung-Russell Diagram \\ 3.4.1. Transformations}

The effective temperatures and bolometric corrections of our stars were determined using photometry and spectral types, if available, or photometry alone, otherwise, in order to place the stars in the H-R diagram. For those stars with spectral types we adopt the calibration of Kilian (1992) for the early B dwarfs and giants, and that of Humphreys \& 


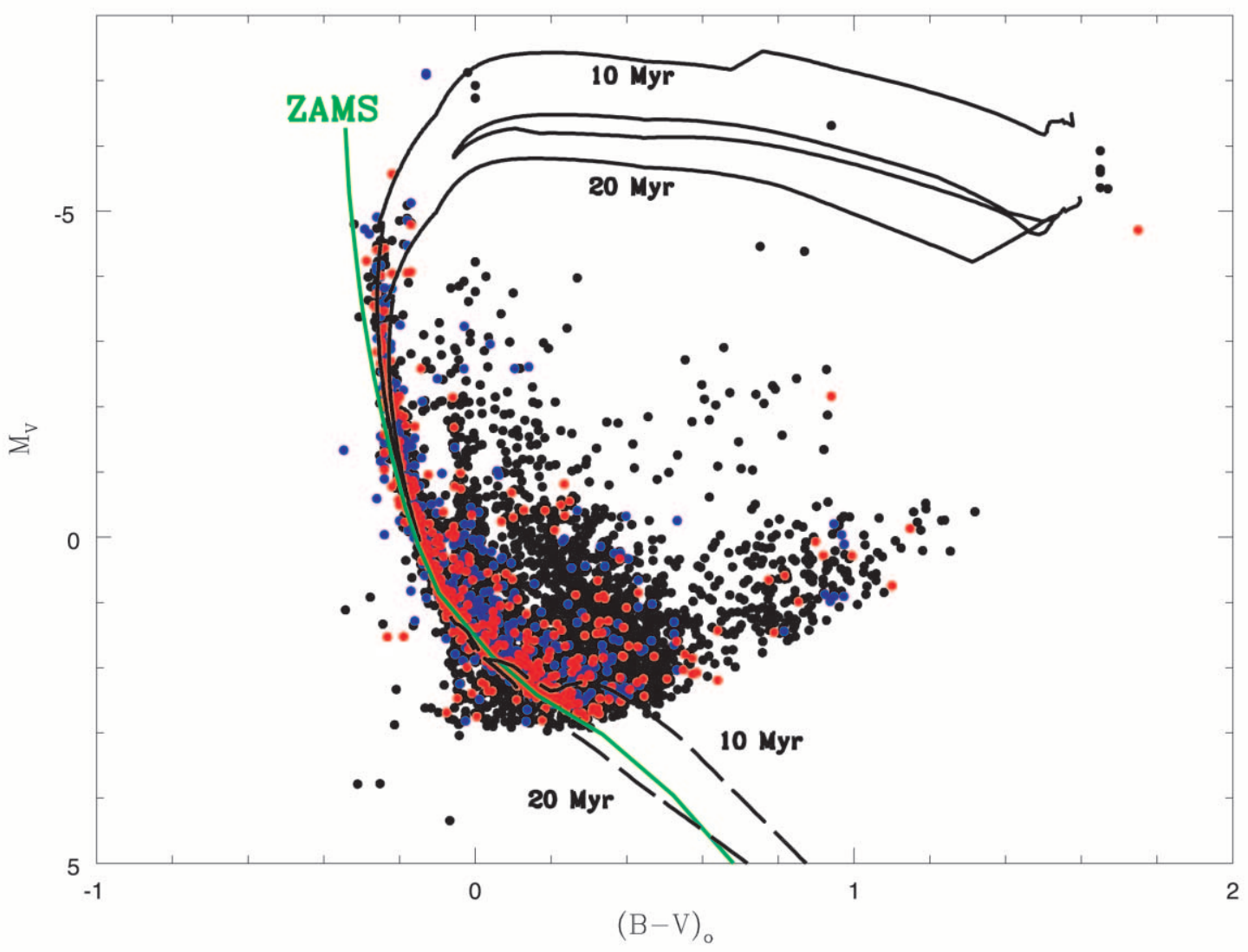

Fig. 6.-Dereddened CMD for stars within $7^{\prime}$ of the center of h (blue) and $\chi($ red $)$ Per are shown now with the zero-age main sequence and post-mainsequence isochrones of 10 and $20 \mathrm{Myr}$ indicated. Corresponding 10 and $20 \mathrm{Myr}$ pre-main-sequence isochrones are shown as dashed lines. The black dots represent the rest of the stars in our full $0.98 \times 0.98$ field.

McElroy (1984) for all other stars. When spectral types were not available, empirically derived relationships were used to transform photometry to $\log T_{\text {eff }}$ and $M_{\text {bol }}$. Effective temperatures were derived for bluest stars $(Q<-0.6)$ using the $Q-\log T_{\text {eff }}$ relationships given by Massey, Waterhouse, \& DeGioia-Eastwood (2000), namely,

$\log T_{\text {eff }}$

$$
= \begin{cases}-0.9894-22.7674 Q-33.0964 Q^{2}-16.19307 Q^{3} & \mathrm{I}, \\ 5.2618+3.4200 Q+2.93489 Q^{2} & \mathrm{III}, \\ 4.2622+0.6452 Q+1.09174 Q^{2} & \mathrm{~V} .\end{cases}
$$

For stars that failed to meet this criterion, we used empirical fits to a combination of observed (Flower 1996) and theoretical (Kurucz 1992) colors and effective temperatures. The former must be used with some caution since there is no reddening correction for what are presumed to be nearby stars. We found

$$
\begin{aligned}
\log T_{\text {eff }}= & 3.9889-0.7950(B-V)_{0}+2.1269(B-V)_{0}^{2} \\
& -3.9330(B-V)_{0}^{3}+3.5860(B-V)_{0}^{4} \\
& -1.5531(B-V)_{0}^{5}+0.2544(B-V)_{0}^{6} .
\end{aligned}
$$

The bolometric correction as a function of effective temperature is that derived by Hillenbrand (1997) for dwarf stars but modified to account for the presence of $\mathrm{M}$ supergiants in our sample by adopting the values in Humphreys
\& McElroy (1984). Thus,

$$
\mathrm{BC}=\left\{\begin{array}{c}
-8.58+8.4647 \log T_{\text {eff }}-1.6125\left(\log T_{\text {eff }}\right)^{2} \\
\log T_{\text {eff }}>4.1 \\
-312.90+161.466 \log T_{\text {eff }}-20.827\left(\log T_{\text {eff }}\right)^{2} \\
4.1>\log T_{\text {eff }}>3.83 \\
-346.82+182.396 \log T_{\text {eff }}-23.981\left(\log T_{\text {eff }}\right)^{2} \\
3.83>\log T_{\text {eff }}>3.55 \\
-2854.91+1590.11 \log T_{\text {eff }}-221.51\left(\log T_{\text {eff }}\right)^{2} \\
3.55>\log T_{\text {eff }} .
\end{array}\right.
$$

\subsubsection{The $H$-R Diagram}

Figure 7 is the resulting H-R diagram. Post-zero-age main sequence evolutionary tracks and isochrones are transformed as above from the $\log T_{\text {eff }}$ and $M_{\text {bol }}$ values calculated by Schaller et al. (1992). All stars with MK spectral classifications of luminosity class I or III and stars earlier than B5 with luminosity class IV or V were placed spectroscopically (filled circles), while most other stars were placed photometrically (open circles). The left panel shows data for the entire imaging area, while the right panel contains only stars within $7^{\prime}$ of the cluster nuclei. No corrections for field star contamination have been applied, and, as was the case for the color-magnitude diagrams (Fig. 5), the H-R diagrams for the central regions of the clusters contain significantly less field star contamination, especially above the 


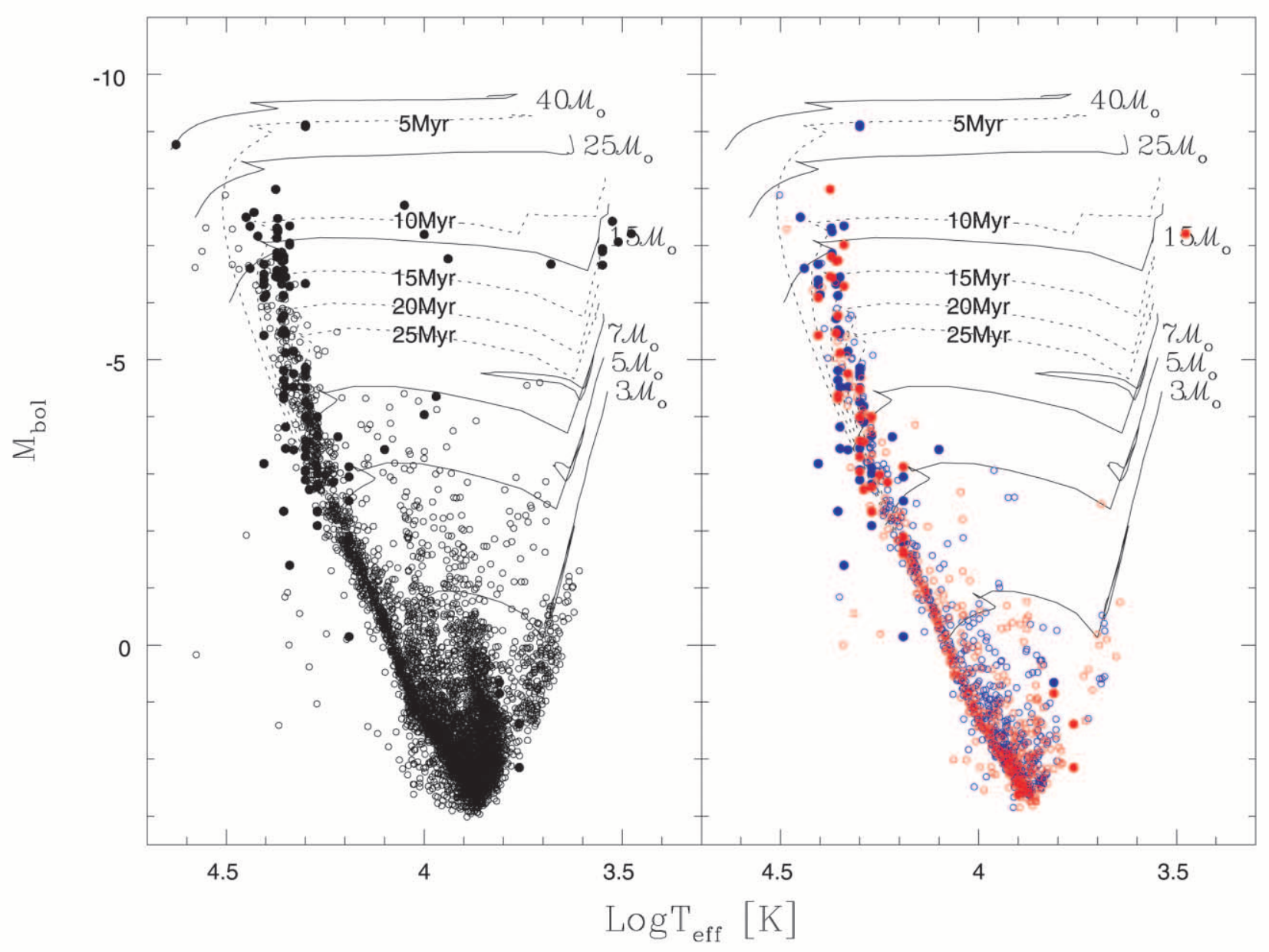

FIG. 7.-H-R diagram of $\mathrm{h}$ and $\chi$ Per are shown. On the left we show all of the data, with filled circles showing the data placed by means of spectroscopy and open circles being the data for which have only photometry. On the right we show only the stars within a $7^{\prime}$ radius of the center of h (blue dots) and $\chi($ red dots).

main sequence. Note the presence of the O-type star HD 14434 in the left panel of Figure 7. It is highly discrepant in age, and, combined with its location outside the cores of the clusters, we dismiss this star as a field star.

From these H-R diagrams we immediately see that the $\mathrm{h} / \chi$ Per clusters are slightly evolved from the zero-age main sequence and that the most massive stars are only $\approx 20-30 M_{\odot}$. The data extend down to about $3 M_{\odot}$ before field star contamination becomes substantial.

\subsubsection{Stellar Ages and the Age Distribution}

For finding ages, we use our dereddened CMD data (Fig. 6) with a grid of isochrones computed at intervals of 0.1 Myr from 5-30 Myr. ${ }^{3}$ We restrict ourselves to only the most luminous stars $\left(M_{V}<-3\right)$, as it is only near and above the turnoff that there is good age information. We filter out the

\footnotetext{
${ }^{3}$ We use the CMD rather than the HRD data to determine ages in order to avoid the quantization problem introduced by spectral types. The ages are very sensitive to $\log T_{\text {eff }}\left[\right.$ or $\left.(B-V)_{0}\right]$, and thus this quantization would introduce a spurious age spread. The spectral types have been employed in the CMD in order to derive $E(B-V)$. In a subsequent section we will use the HRD to derive the mass function. The masses are primarily sensitive to an accurate determination of $M_{\mathrm{bol}}$, which we expect to be better determined using the bolometric corrections determined from spectral types.
}

obvious foreground contaminants, e.g., $(B-V)_{0}>-0.2$ for $-3>M_{V}>-5$. We cannot use the red supergiants (RSGs) for our age determinations, unfortunately, since the evolutionary tracks do not actually extend that far to the red; we will note, though, that the location of the RSGs in the CMD are consistent with the ages that we would derive were we to extrapolate the isochrones. For each of our clusters we find essentially identical ages: 12.8 and $12.9 \mathrm{Myr}$ for $\mathrm{h}$ and $\chi$, respectively. The formal errors of the mean on these determinations are $1 \mathrm{Myr}$, and the scatter is $\sim 5 \mathrm{Myr}$; the latter is dominated by observational errors at the $<0.01 \mathrm{mag}$ level.

We do not find evidence for multiple distinct episodes of star formation despite the remarkable similarities between our dereddened CMD and Wildey's (1964). We believe the difference in interpretation occurs because Wildey in his original analysis did not consider the possibility of field star contamination from $\mathrm{G}$ and $\mathrm{K}$ giants seen to large distances through the Galaxy. It is clear from the right panel of Figure 7 that when just the cluster nuclei are considered any apparent branching in the H-R diagram is significantly diminished. We do find several high-mass stars with uncharacteristically young ages as compared to the rest of the cluster. However, in most cases these stars are either not in the central regions of the clusters or their spectroscopically derived distance is inconsistent with their being cluster members. 
Although our data are consistent with the $\mathrm{h} / \chi$ stars having formed in a single burst, we cannot rule out other scenarios. For instance, if the primary burst of star formation has occurred at $13 \mathrm{Myr}$, with a smaller, secondary burst at $10 \mathrm{Myr}$, we would very likely not discern this in our CMD. There would be few high-mass stars, and the lower mass stars would be indistinguishable from their 13 Myr counterparts.

\subsubsection{Stellar Masses, the Mass Function, and Mass Segregation}

Masses are inferred for individual stars by interpolating between the mass tracks on the H-R diagram. By counting the number of stars found in each mass bin, we derive the present-day mass function (PDMF). To the extent that star formation may be coeval, this is equivalent to the initial mass function (IMF), except for the depopulation of the highest mass bin.

In order to minimize the effect of field star contamination, PDMFs have been constructed only for the two regions within $7^{\prime}$ of the cluster cores. In addition, we exclude a few stars found redward of the main sequence and presumed to be foreground contaminants, by eliminating stars in the region constrained between $M_{\text {bol }}<-20.5 \log T_{\text {eff }}+82.5$ and $M_{\text {bol }}>-5$. We used a lower mass cutoff of $4 M_{\odot}$ below which field and pre-main-sequence star contamination dominates. At the high-mass end, we expect that evolution through the supernova phase will have depleted stars above $\sim 15-20 M_{\odot}$, and so we have used only the mass bins below this to compute the slope of the IMF. We combine all of the higher mass stars into one mass bin. Following Scalo (1986), we define the quantity $\xi$ as the number of stars per mass bin divided by the difference in the base-10 logarithms of the upper and lower bin masses and also by the surface area in kiloparsecs. The run of $\log \xi$ with $\log$ mass then provides the slope, $\Gamma$, of the IMF/PDMF. Values for the number of stars and for $\xi$ are given in Table 3 .

Figure 8 shows PDMFs in the $4-16 M_{\odot}$ range for stars within $7^{\prime}$ of the cluster centers. Error bars are based on $\pm \sqrt{N}$ statistics. We obtain values of $\Gamma=-1.36 \pm 0.20$ for $\mathrm{h}$ Per and $\Gamma=-1.25 \pm 0.23$ for $\chi$ Per. Within the errors of our fits, both slopes are in good agreement with each other and also with the Salpeter value of $\Gamma=-1.35$. This result can be compared with what is known of the IMF in other young $\mathrm{OB}$ associations and clusters, where a weighted average yields $\Gamma=-1.1 \pm 0.1$ for the Milky Way and $\Gamma=-1.3 \pm 0.1$ for the LMC/SMC (Massey 1998b). Thus, an IMF slope of $\Gamma=-1.3 \pm 0.2$ for $h$ and $\chi$ is in no way unusual.

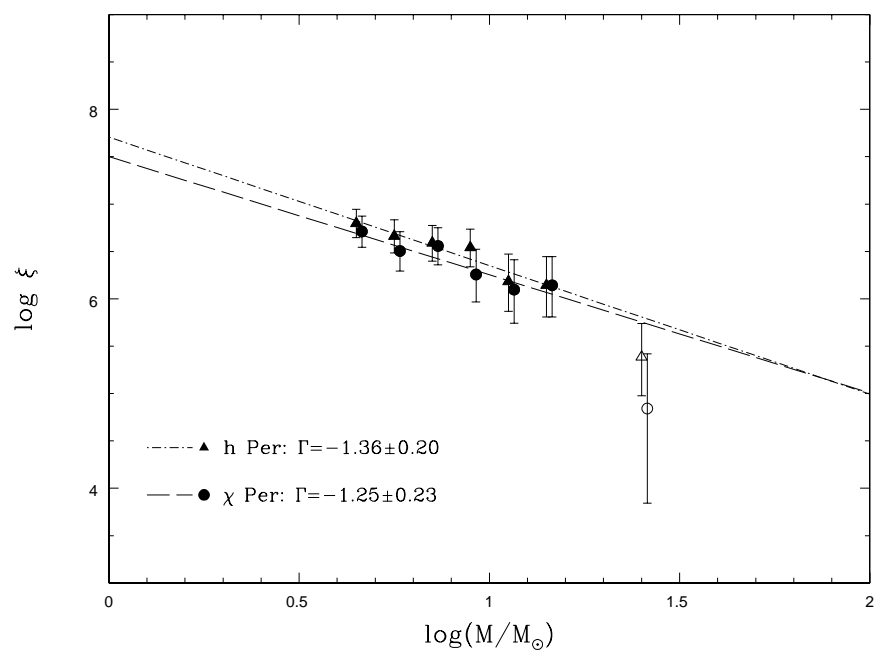

FIG. 8.- Initial mass function is shown for the two clusters. Open symbols indicate an incomplete bin.

Based on extrapolation of the measured PDMFs to 120 $M_{\odot}$, we estimate that $\sim 40$ supernovae have occurred in the past in the central regions of the $\mathrm{h} / \chi$ Per clusters. Assuming a constant mass function from $1-120 M_{\odot}$, we can estimate the total stellar mass within each of the cluster centers down to $1 M_{\odot}$. We find values of 3700 and $2800 M_{\odot}$ for $\mathrm{h}$ Per and $\chi$ Per, respectively. This is about 8-10 times that of the mass in greater than $1 M_{\odot}$ stars in the younger Orion Nebula cluster $\left(\mathrm{ONC} ; \sim 450 M_{\odot}\right)$ or the older Pleiades $\left(\sim 320 M_{\odot}\right)$. For comparison, a "supercluster" like R136 in the LMC has a mass of roughly $(3-4) \times 10^{4} M_{\odot}$ in greater than $1 M_{\odot}$ stars (Hunter et al. 1996), about a factor of 10 greater than either $\mathrm{h}$ or $\chi$ and a factor of almost 100 greater than Orion or the Pleiades.

In Figure 9 we explore the evidence for concentration and mass segregation in the two clusters. In doing so, we consider only those stars satisfying our criteria for inclusion in the PDMF. In viewing these panels it should be noted that the $2 \sigma$ surface density contour in the spatial distribution of stars occurs at radii of $\sim 7^{\prime}$ for both $\mathrm{h}$ Per and $\chi$ Per. The top and middle panels of Figure 9 show that inside of $7^{\prime}$, both the mass surface density and the number surface density begin to rise noticeably above the field star surface density and then steepen considerably at $\sim 3^{\prime}$. The increase in density at smaller cluster radii is evidence of higher central concentration.

The histograms of the total mass per square parsec as a function of radial distance (Fig. 9, top panels) show that $\mathrm{h}$ Per is about twice as dense at its core compared to $\chi$ Per. This occurs both because $\mathrm{h}$ Per has $\sim 25 \%$ more stars at its center (as can be seen in the middle panels of Fig. 9) and because it contains several high-mass $\left(>30 M_{\odot}\right)$ B supergiants. However, the density profile of $\mathrm{h}$ Per falls off more rapidly than that of $\chi$ Per, and the two clusters are roughly equivalent in mass density at a radius of $\sim 3^{\prime}$.

The bottom panels of Figure 9 show the average mass as a function of radial distance from the cluster centers. For $h$ Per, we find a significant gradient inside of $\sim 7^{\prime}$ in the mean mass versus radial distance, suggestive of mass segregation. The data for $\chi$ Per is less convincing, yet we still find the mean stellar mass to be higher by $\sim 1.5-2 \sigma$ within the central $1^{\prime}$. This phenomenon has been claimed with varying 

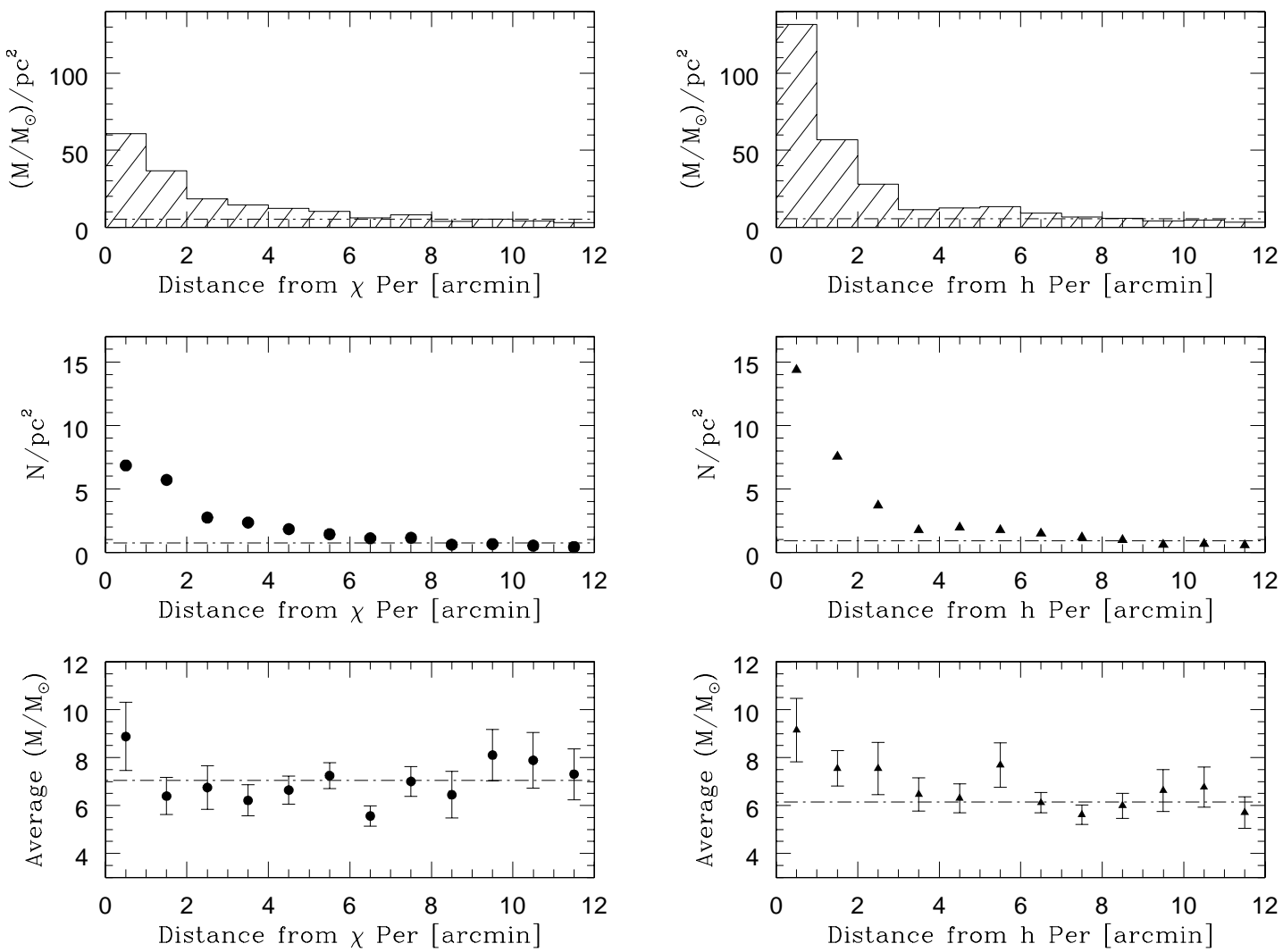

FIG. 9.- Total mass per unit area (top panels), the number of stars per unit area (middle panels), and the average stellar mass per unit area (bottom panels) are shown as a function of radial distance for $\chi \operatorname{Per}(l e f t)$ and $\mathrm{h}$ Per $(r i g h t)$. The data have been binned in $1^{\prime}$ rings from the respective cluster centers. Horizontal dashed lines indicate the average of the values from $6^{\prime}-12^{\prime}$. Within the $2 \sigma$ surface density enhancements $\left(r=7^{\prime}\right)$, there is clear evidence in both h and $\chi$ Per for central concentration within $3^{\prime}$ (top and middle panels) and also some evidence for mass segregation within $1^{\prime}-2^{\prime}$ (bottom panels).

degrees of strength in other open clusters in the Galaxy (e.g., the Orion Nebula cluster [Hillenbrand \& Hartmann 1998 and references therein]) and in the Magellanic Clouds (e.g., R136 [Hunter et al 1995] and NGC 1805 and NGC 1818 [deGrijs et al 2002]). However, unlike their younger counterparts, the mean mass gradient in $\mathrm{h} / \chi$ Per may not be primordial, i.e., associated with the formation of the clusters. Assuming a velocity dispersion of $\sigma_{v} \approx 3 \mathrm{~km} \mathrm{~s}^{-1}$ and a $7^{\prime}(4.79 \mathrm{pc})$ cluster radius, we estimate a crossing time of $\sim 1.56 \mathrm{Myr}$ for each of the cluster nuclei. Given that the clusters are $\sim 13 \mathrm{Myr}$ old, the age $/ t_{\text {cross }} \approx 8$, and hence dynamical relaxation may indeed play some part in the observed mass segregation.

\section{DISCUSSION}

\subsection{Comments on the Uniqueness of $h / \chi$ Per}

The $\mathrm{h} / \chi$ Per clusters are separated by about $30^{\prime}$ on the sky, equal to $20 \mathrm{pc}$, and are located $\sim 3.5$ or $140 \mathrm{pc}$ out of the plane of the Galaxy. They are thus similar to but larger and more massive than the younger, closer Orion Nebula cluster and NGC 2024 pair, which are separated by $\sim 32 \mathrm{pc}$ and located $\sim 150 \mathrm{pc}$ out of the plane, or the IC 348 and NGC 1333 pair, $\sim 21$ pc from each other and $\sim 122$ pc from the plane. As noted above, however, the massive star content of $\mathrm{h} / \chi$ Per is more than an order of magnitude higher than it is in these regions. Other suggested coeval doublecluster systems include the older SL 538/SL 537, SL 353/SL
349, SL 387/SL 385, NGC 1971/NGC 1972, and NGC 1850 pairs (e.g., Dieball \& Grebel 1998, 2000a, 2000b and references therein), all in the LMC (see Bhatia \& Hatzidimitriou 1988 and Hatzidimitriou \& Bhatia 1990 for other LMC and SMC candidates), and the young NGC 206 (van den Bergh 1966; see also Massey, Armandroff, \& Conti 1986) in M31. The range in scale of double-cluster formation may extend, therefore, from clusters of individual size ranging from a few parsecs up to a few hundred parsecs in diameter. In the younger of these double clusters, for example, the ONC/NGC 2024 pair, the stellar and cluster dynamics are still dominated by molecular gas, and the clusters are at best only marginally/loosely bound once the gas dissipates, unlike $\mathrm{h} / \chi$ Per, which have survived as bound clusters for $\sim 10 \mathrm{Myr}$ after gas dissipation. At present, however, kinematic studies of the $\mathrm{h} / \chi$ Per cluster motions relative to one another are needed in order to decipher whether the $\mathrm{h} / \chi$ Per clusters are a true binary system or merely reflective of synchronized star formation on larger size scales.

Despite the above suggestion that double-cluster formation may be fairly common, we now argue that $\mathrm{h} / \chi$ Per are nearly unique. They are remarkably similar clusters insofar as we find their distances, reddenings, ages, IMF slopes, and physical sizes to be indistinguishable. The stellar density of $\mathrm{h}$ Per, however, is a factor of 2 higher than that of $\chi$ Per, and its total mass about one-third more. Independent of whether this single difference is considered or ignored, the $\mathrm{h} / \chi$ Per system evokes the word "unique" when considered 
in the context of the Galaxy. The system is, after all, commonly known as the double cluster. While the LMC may contain a high proportion of double clusters that are coeval, these systems show a wide range in total mass ratio and size ratio (see, e.g., Leon, Bergond, \& Vallenari 1999).

\subsection{Comments on the Relationship between $h / \chi$ Per and Per OBI}

The $\mathrm{h} / \chi$ Per clusters are often described as the core of the Per OB1 association, located in the Perseus spiral arm at a distance of $2.3 \mathrm{kpc}$ (Humphreys 1978; Ruprecht 1966). A similar relationship between clusters and associations may hold in other cases, such as the pair of open clusters IC 1805/IC 1848 and Cas OB6, seen in projection only $5^{\circ}$ from $\mathrm{h} / \chi$. Garmany \& Stencel (1992) question the physical relation between $\mathrm{h} / \chi$ Per and the Per OB1 association, other than being located along the same line of sight and in the same spiral arm, which is nearly perpendicular to our line of sight in the direction $l \approx 135^{\circ}$. That we see $\mathrm{h} / \chi$ Per projected in a field star distribution that is, to within a factor of $30 \%$, at the same distance as the clusters complicates discussion of the cluster/OB association relationship. This discussion is further complicated by the similarity in age between the Per OB1 field population and the $\mathrm{h} / \chi$ Per clusters.

Per OB1 is particularly notable for containing the largest number of RSGs among the associations whose high-mass members were cataloged (e.g., Blanco 1955; Humphreys 1970; Garmany \& Stencel 1992; see also Bidelman 1947b), as well as a substantial number of A- and B-type supergiants (Bidelman 1943). RSGs are visible only for a narrow range of ages between 10-25 Myr at the completeness limit of our photometry and considering the distance to the Perseus spiral arm. Thus, it is difficult to reconcile whether the red supergiants at large projected distances from the $\mathrm{h} / \chi$ Per cluster (Fig. 7, left panel, filled circles at $\log T_{\text {eff }}=3.5$ ) are part of the field or the result of past ejection from the $h / \chi$ Per cluster core regions. Ejection of massive stars from a dense cluster can occur for particular binary and system orbital parameter combinations but requires that the cluster is mass segregated at very young ages, e.g., at or before the time of gas expulsion (Kroupa 2002). Populating the entirety of the Per OB association with stars ejected from the centers of $h / \chi$ Per is unlikely, though the effect may be as large as $10 \%-30 \%$ by the present cluster ages. The double nature of the cluster may also be important for stellar dynamics considerations.

Along the main sequence of the H-R diagram we find reasonable agreement between the cluster and field populations, again because of the similarity in distance and age of the massive star population. But this does not prove physical association between the clusters and the field or association. Even kinematic information would be of limited use in this debate, given the magnitude of the effect compared to achievable errors.

\subsection{Future Work}

At an age of $13 \mathrm{Myr}$, the $\mathrm{h} / \chi$ Per clusters occupy a particularly interesting age range for investigations of circumstellar disk dissipation and of stellar angular momentum evolution. The evolutionary paths of these phenomena are very poorly understood between the age ranges of wellstudied star-forming regions $(<1-3 \mathrm{Myr})$ and the nearest open clusters (IC 2602 and IC 2391 at $50 \mathrm{Myr}, \alpha$ Per at 80 Myr, and the Pleiades at 120 Myr). Despite the larger distance relative to some of these other well-studied open clusters, investigations of the lower mass $\left(<3 \quad M_{\odot}\right)$ stellar content of $\mathrm{h} / \chi$ Per is therefore of great interest. Substantial field star contamination will complicate this issue and require selection techniques such as X-ray or $\mathrm{H} \alpha$ emission or photometric variability to separate young active candidate cluster members from the Galactic plane foreground/ background in a photometric survey.

\section{SUMMARY}

We have studied the $\mathrm{h}$ and $\chi$ double cluster using modern imaging and spectroscopic techniques. We find that the two clusters have indistinguishable reddenings $[E(B-V)=0.56]$ and distances $\left[(m-M)_{0}=11.85\right]$, values consistent with those cited in the literature. Especially impressive is that these conclusions are identical to those of Wildey (1964), whose data we find holds up extremely well against the current analysis.

Where we differ with previous studies such as Wildey's (1964) is in our recognition of the significant effect that field star contamination has on the determination of cluster ages. Inclusion of foreground younger stars and GK giants can easily lead to apparent branching in the H-R diagram, which has been misinterpreted in the past as an age spread. We find mean ages of 12.8 Myr for each of the two clusters and no evidence for multiple epochs of star formation.

The present-day mass function yields a slope consistent with that found in other well-studied Galactic OB associations and clusters $(\Gamma \sim-1.1 \pm 0.1$; see Massey $1998 \mathrm{~b})$ and is essentially Salpeter $(\Gamma=-1.35)$. In addition, we do find some evidence of mass segregation. The total masses are $3700 M_{\odot}$ for h Per and $2800 M_{\odot}$ for $\chi$ Per, for stars with greater than $1 M_{\odot}$.

Our interest in $\mathrm{h}$ and $\chi$ Per traces back to several conversations with Stephen Strom, who remarked at least once how hard it was to understand a 50 Myr age spread, lamenting that "if we do not understand star formation in $\mathrm{h}$ and $\chi$ Per, then where do we understand it?" We hope that we have partially addressed this concern. It is a pleasure to thank Michael Meyer and John Carpenter for work they did in measuring coordinates of the $h$ and $\chi$ stars, which we used for spectroscopy prior to our CCD imaging efforts. We also acknowledge help and advice from George Jacoby in obtaining the Mosaic data. C. L. S. became involved in this project as a Research Experiences for Undergraduates participant in 1999, and her efforts were supported first by the National Science Foundation (NSF) under grant 99-88007 to Northern Arizona University and more recently by an NSF graduate research fellowship. We dedicate this paper to the memory of Bob Wildey, whose Ph.D. thesis on the subject of $\mathrm{h} / \chi$ Per should be required reading for all students of Galactic astronomy. 
Appenzeller, I. 1967, PASP, 79, 102

Balona, L. A., \& Feast, M. W. 1975, MNRAS, 172, 191

Balona, L. A., \& Shobbrook, R. R. 1984, MNRAS, 211, 375

Bernasconi, P. A. 1996, A\&AS, 120, 57

Bhatia, R. K., \& Hatzidimitriou, D. 1988, MNRAS, 230, 215

Bidelman, W. P. 1943, ApJ, 98, 61 -1947a, AJ, 52, 121 . 1947b, ApJ, 105, 492

Blaauw, A. 1964, ARA\&A, 2, 213

Blanco, V. M. 1955, ApJ, 122, 434

Conti, P. S. 1988, in O Stars and Wolf-Rayet Stars, ed. P. S. Conti \& A. B. Underhill (NASA SP-497), 121

Crawford, D. L., Glaspey, J. W., \& Perry, C. L. 1970, AJ, 75, 822

de Grijs, R., Gilmore, G. F., Johnson, R. A., \& Mackey, A. D. 2002, MNRAS, 331, 245

Dieball, A., \& Grebel, E. K. 1998, A\&A, 339, 773 2000a, A\&A, 358, 144 2000b, A\&A, 358, 897

FitzGerald, M. P. 1970, A\&A, 4, 234

Flower, P. 1996, ApJ, 469, 355

Garmany, C. D., \& Stencel, R. E. 1992, A\&AS, 94, 211

Hatzidimitriou, D., \& Bhatia, R. K. 1990, A\&A, 230, 11

Hillenbrand, L. A. 1997, AJ, 113, 1733

Hillenbrand, L. A., \& Hartmann, L. W. 1998, ApJ, 492, 540

Humphreys, R. M. 1978, ApJS, 38, 309

Humphreys, R. M., \& McElroy, D. B. 1984, ApJ, 284, 565

Humphreys, R. W. 1970, ApJ, 160, 1149

Hunter, D. A., O'Neil, E. J., Lynds, R., Shaya, E. J., Groth, E. J., \& Holtzman, J. A. 1996, ApJ, 459, L27

Hunter, D. A., Shaya, E. J., Holtzman, J. A., Light, R. M., O’Neil, E. J., \& Lynds, R. 1995, ApJ, 448, 179

Jacoby, G. H., Hunter, D. A., \& Christian, C. A. 1984, ApJS, 56, 257

Jaschek, C., \& Jaschek, M. 1987, The Classification of Stars (Cambridge: Cambridge Univ. Press)

Johnson, H. L., \& Morgan, W. W. 1955, ApJ, 122, 429

Keller, S. C., Grebel, E. K., Miller, G. J., \& Yoss, K. M. 2001, AJ, 122, 248

\section{REFERENCES}

Kilian, J. 1992, A\&A, 262, 171

Kroupa P. 2002, habilitation thesis, Univ. Kiel

Kurucz, R. 1992, in The Stellar Populations of Galaxies, ed. B. Barbuy \& A. Renzini (Dordrecht: Kluwer), 225

Landolt, A. U. 1992, AJ, 104, 340

Leon, S., Bergond, G., \& Vallenari, A. 1999, A\&A, 344, 450

Marco, A., \& Bernabeu, G. 2001, A\&A, 372, 477

Massey, P. 1998a, in Stellar Astrophysics for the Local Group, ed. A. Aparcicio, A. Herrero, \& F. Sanchez (Cambridge: Cambridge Univ. Press), 95

.1998b, in ASP Conf. Ser. 142, The Stellar Initial Mass Function, ed. G. Gilmore \& D. Howell (San Francisco: ASP), 17

Massey, P., Armandroff, T. E., \& Conti, P. S. 1986, AJ, 92, 1303

Massey, P., Johnson, K. E., \& DeGioia-Eastwood, K. 1995, ApJ, 454, 151

Massey, P., Waterhouse, E., \& DeGioia-Eastwood, K. 2000, AJ, 119, 2214

Morgan, W. W., Code, A. D., \& Whitford, A. E. 1955, ApJS, 2, 41

Morton, D. C. \& Adams, T. F. 1968, ApJ, 151, 611

Oosterhoff, P. T. 1937, Ann. Sternw. Leiden, 17, 1

Ruprecht, J. 1966, Bull. Astron. Inst. Czechoslovakia, 17, 33 (erratum 17, 98)

Scalo, J. M. 1986, Fundam. Cosmic Phys., 11,

Schaller, G., Schaerer, D., Meynet, G., \& Maeder, A. 1992, A\&AS, 96, 269

Schild, R. 1965, ApJ, 142, 979 1967, ApJ, 148, 449

Schmidt-Kaler, T. 1982, in Astronomy and Astrophysics, Vol. 2b, Stars and Star Clusters, ed. K. Schaifers \& H. H. Voigt (Berlin: Springer), 454 Slettebak, A. 1968, ApJ, 154, 933

Tapia, M., Roth, M., Costero, R., \& Navarro, S. 1984, Rev. Mexicana Astron. Astrofis., 9, 65

Valdes, F. 1998, Guide to the NOAO Mosaic Data Handling Software (NOAO)

van den Bergh, S. 1966, AJ, 71, 219

Vogt, N. 1971, A\&A, 11, 359

Waelkens, C., et al. 1990, A\&AS, 83, 11

Walborn, N. R., \& Fitzpatrick, E. L. 1990, PASP, 102, 379

Wildey, R. L. 1964, ApJS, 8, 439 